\title{
Analysis of Dynamic Parameters of a Linear Positioning Table System
}

\author{
Vytautas TURLA*, Artūras KILIKEVICIUS**, Mindaugas JUREVICIUS***, Antanas \\ FURSENKO $* * * *$, Kristina KILIKEVICIENE*****, Gulnara ZHETESSOVA******, Olga \\ ZHARKEVICH*********
}

*Vilnius Gediminas Technical University, Basanaviciaus 28, 03224 Vilnius, Lithuania, E-mail: vytautas.turla@vgtu.lt; **Vilnius Gediminas Technical University, Basanaviciaus 28, 03224 Vilnius, Lithuania,

E-mail: arturas.kilikevicius@vgtu.lt;

***Vilnius Gediminas Technical University, Basanaviciaus 28, 03224 Vilnius, Lithuania,

E-mail: mindaugas.jurevicius@vgtu.lt;

****Vilnius Gediminas Technical University, Basanaviciaus 28, 03224 Vilnius, Lithuania,

E-mail: antanas.fursenko@vgtu.lt;

*****Vilnius Gediminas Technical University, Basanaviciaus 28, 03224 Vilnius, Lithuania,

E-mail: kristina.kilikeviciene@vgtu.lt;

******Karaganda State Technical University, Nursultan Nazarbayev Avenue 56, 100027 Karaganda, Kazakhstan,

E-mail: zhetesova@mail.ru;

*******Karaganda State Technical University, Nursultan Nazarbayev Avenue 56, 100027 Karaganda, Kazakhstan,

E-mail: zharkevich82@mail.ru

cross $^{\text {ref }}$ http://dx.doi.org/10.5755/j01.mech.26.5.24866

\section{Introduction}

Demands for machining and metrology over a relatively large range have recently increased in a variety of industries and sciences such as aerospace, electronics, energy, and astronomy [1], [2] and [3]. In particular, such demands result in the development of innovative table systems which achieve long range positioning with a nanometer resolution [4]. Although many studies on ultra-precision positioning table system have been performed so far, there were only few studies on table system which could achieve positioning with sub-nanometer resolution in a long travel range [5].

In his work, scientist Roy S. Colby presents the results of acoustic research [6]. In the paper, he points out how the levels of vibrations and sound depend on the rotational speed and the frequency of vibrations. The goal of the work carried out by Eduardo Rubio and Juan C. Jauregui was an establishment of the condition of a mechanism required for avoiding random breakdowns of such a mechanism upon applying the methods of periodic monitoring and analysis of vibrations [7]. In scientific papers (by Ching Yuan Lin, Jui Pin Hung and Tzuo Liang Lo), dynamic characteristics of vertical milling machines were established and their structural characteristics were examined $[8,9]$. The said works are relevant because guides and other structural elements used in CNC milling machines are assembled of different elements connected with each other by threaded junctions. For linear shifts, slideway bearings with carriage (usually with grooves with balls in the latter) are used. In the work on a dynamic analysis of machine [10], the influence of a preload setting by a ball screw on the axle stiffness was calculated upon applying Hertz contact theorem. The effect of the preload torque on the connection of the screw, when dynamic stiffness and dampness of linear guides are identified according to optimum models was examined. The same problem is important in investigation of movement trajectories of robot manipulators [11-18].
In the presented paper, an analysis of dynamic parameters of a linear positioning table system upon applying the theory of random functions is carried out.

The temporal dependence structure of a stationary stochastic process characterized by the auto-covariance function, or equivalently by its Fourier transform was described by Proietti and Luati [19]. The spectral density functions are defined in scientific work by Siljak and Subasi [20]. Dutkay and Jorgensen described Fourier series fractals [21]. Model-based filtering method for data reconciliation, introduced by Narisimhan and Jordache [22], applies modest process models to advance the precision and reliability of gauged data. In favourable observation circumstances, data reconciliation also enables the evaluation of ungauged process data. These capabilities are appreciated for process productions since in many practical cases strategic data are gauged only with imperfect accuracy or just are not gauged because of mechanical or financial restraints. Data reconciliation has been used for a great quantity of processes as summarized by Narasimhan and Jordache [22]. Other topics [23, 24] are linked to the data reconciliation, such as sampling fault and reconciliation weighing factor assessment, resigned value correctness estimation, use of resigned data to determine and to define plant performance directories, such as focus score and recapture.

The reconciliation of process fathoming subject to linear restraints includes definition of the least weighted sum of squares of modifications to the measurements. Linear values settlement by maximizing the data entropy to get probability of value spreading with the lowest integration of former knowledge was reformulated in the paper by Crowe [23]. Two occasions were deliberated by researchers, the first, with only the boundaries on the data being quantified and, the second, with the variance-covariance vector of the data being quantified additionally. The first occasion presents an instrument for executing data reconciliation in the deficiency of information on the variance-covariance matrix 
of the data. In the second case, reconciliation using maximum probability is formally identical to the conventional the least-squares solution [22]. The least-prejudiced likelihood distribution is an abbreviated normal distribution, which for reasonably accurate data matches with the multivariate normal distribution.

Different data reconciliation techniques proposed are based on various assumptions concerned with the process dynamics and depending on the subsequent application of the reconciled data [23]. Steady-state data reconciliation is generally used to assess the fundamental normal regime of a plant in applications, such as decrease accounting, on process audit, or survey analysis presented by Poulin et al. [24]. Then, progressive process control, error recognition algorithms, and real-time optimization need the assessment of right dynamic states of a process, and are usually coupled to dynamic data reconciliation. Nevertheless, different methods could be used for reconciliation of dynamic data. The selected process model influences on the range of filtering algorithm complexity. It could vary from a modest mass conservation restraint sub-model to a comprehensive causal dynamic model. The choice of the most proper procedure effects on a compromise between modelling efforts, required to progress and adapt the observer and to improve the evaluation correctness.

Researchers Puigjaner and Heyen [25] have reasoned the features of steady-state observers and related problems, such as steady-state definition and redundancy analysis (RDA), as well as substantial fault finding-out. Researchers Narasimhan, Jordache and Crowe considered steady-state data reconciliation which functional on a realtime basis $[22,23]$. They have determined that, despite the solution's simplicity, the assessment precision maybe be less than measurement accuracy itself depending on plant dynamics. The proposed method as modified stationary one that has progress of the correlation of node imbalances to advance assessment performances.

Wavelet analysis recognized recently as a tool for important applications in time series, function estimation and image analysis. According to the recently developed wavelet methods, the fundamentals are not yet widely understood, and the guidance on their practical application is hard to find. Wavelets are an increasingly and widely used tool in numerous applications of signal and image processing. Adjustments in distant detecting include the grouping of different resolution pictures, picture compression, and the delivery of frame recognition methods. Researcher Horgan [26] has reviewed the basic ideas of wavelets in order to represent the information in signals, such as time series and images, and described how the wavelet shrinkage is applied to smooth these signals. This was illustrated by the application to a synthetic aperture radar image.

Researchers Ekstrom and McEwen [27] improved adaptive box-filtering measures to eliminate random bit faults (pixel values in case if they are not related to the picture) and even noise data, i.e. pixels of the picture related with an adulterant or multiplicative noise element. For both cases, they used the standard deviation of these pixels within a local box surrounding each pixel, after that they used adaptive filters. This method effectively reduced speckle in radar pictures without removing important details.

The assessments of the cross-covariance function of two numerical data massifs of the distortion signals or the evaluations of auto-covariance function of a distinct massif calculated by alteration of the numerical vibration massifs into vectors. For treatment of the numerical signals, discrete Fourier transform described by researchers Ekstrom, McEwen [27] and the theory of wavelets were applied in scientific papers of Antoine [28], Berczynski and Wroblewski [29].

\section{Theoretical and experimental research}

\subsection{A covariance model of parameters of vibration signals}

The linear positioning table system has been investigated in two operating modes. In the first mode, lubricant Loctite 8103 was used and in the second mode, lubricant Braycote $601 \mathrm{EF}$ was used. In each of the said operating modes, four speeds of the carriage of positioning table were used: $0.25 ; 1.25 ; 2.5$; and $3.75 \mathrm{~mm} / \mathrm{s}$, respectively.

For a theoretical model, we'll apply an assumption that errors in measuring the intensity of digital vibration signals of the system are random and possibly systematic.

In each vector of the vibration intensity measurement data array, the trend of the measurement data of that vector is eliminated. As one of parameters, the time interval of electromagnetic spread is used.

We'll consider the random function (formed according to the data arrays of measuring the intensity of digital vibration signals) a stationary function (in a broad sense), i.e. its average value $M\{\varphi(t)\} \rightarrow$ const, and the covariance function depends only on the difference of arguments $-K_{\varphi}(\tau)$. The auto-covariance function of a single data array or the cross-covariance function of two data arrays $K_{\varphi}(\tau)$. will be expressed as follows [30]:

$$
K_{\varphi}(\tau)=\frac{1}{T-\tau} \int_{0}^{T-\tau} \delta \varphi_{1}(u) \delta \varphi_{2}(u+\tau) d u,
$$

here: $\delta \varphi_{1}(u), \delta \varphi_{1}(u+\tau)$ - the centered estimates of measuring the intensity of vibration signals; $u$-vibration parameter; $\tau=k \cdot \Delta$ - variable quantization interval; $k$ - the number of units of measurement; $\Delta$ - the value of a unit of measurement; $T$ - time.

The estimate $K_{\varphi}(\tau)$ of the covariance function according to the available data on measurement of vibration parameters is calculated as follows:

$$
K_{\varphi}^{\prime}(\tau)=K_{\varphi}^{\prime}(k)=\frac{1}{n-k} \sum_{i=1}^{n-k} \delta \varphi_{1}\left(u_{i}\right) \delta \varphi_{2}\left(u_{i+k}\right)
$$

here: $n$ - the total number of discrete intervals.

The formula (2) may be applied in a form of an auto-covariance function or a cross-covariance function. When the function is an auto-covariance function, the arrays $\varphi_{1}(u)$ and $\varphi_{2}(u+\tau)$ are parts of single arrays and when the function is a covariance function, they are two different arrays.

The estimate of a normed covariance function is:

$$
R_{\varphi}^{\prime}(k)=\frac{K_{\varphi}^{\prime}(k)}{K_{\varphi}^{\prime}(0)}=\frac{K_{\varphi}^{\prime}(k)}{\sigma_{\varphi}^{\prime 2}},
$$


here: $\sigma_{\varphi}^{\prime}-$ the estimate of the standard deviation of a random function.

For elimination of the trends of columns in the $i$-th digital array of measurements, the following formulas are applied:

$$
\delta \varphi_{i}=\varphi_{i}-e \cdot \bar{\varphi}_{i}^{T}=\left(\delta \varphi_{i 1}, \ldots, \delta \varphi_{i m}\right),
$$

here: $\delta \varphi_{i}$ is the $i$-th array of reduced values of the $i$-th digital array where a trend of columns is eliminated; $\varphi_{i}$ is the $i$-th array of vibration intensity; $e$ is a unit vector with the sizes ( $n \times 1) ; n$ is the number of lines in the $i$-th array; $\bar{\varphi}_{i}$ is the vector of averages of the columns in the $i$-th array; $\delta \varphi_{i j}$ is the $j$-th column (vector) of the reduced values in the $\underline{i \text {-th }}$ array.

The vector of the arithmetic mean of columns of the $i$-th array is calculated according to the following formula:

$$
\bar{\varphi}_{i}^{T}=\frac{1}{n} e^{T} \cdot \varphi_{i}=\frac{1}{n} \varphi_{i}^{T} \cdot e . .
$$

The realization of the random function of the $j$-th column in the $i$-th array of vibration intensity measurement data in the vectorial form is expressed as follows:

$$
\delta \varphi_{i j}=\left(\delta \varphi_{i j, 1}, \ldots, \delta \varphi_{i j, m}\right)
$$

The estimate of the covariance matrix of vibration intensity in the i-th array is expressed as follows:

$$
K^{\prime}\left(\delta \varphi_{i}\right)=\frac{1}{n-1} \delta \varphi_{i}^{T} \delta \varphi_{i}
$$

The estimate of the covariance matrix of two arrays of vibration intensity expressed as follows:

$$
K^{\prime}\left(\delta \varphi_{i}, \delta \varphi_{j}\right)=\frac{1}{n-1} \delta \varphi_{i}^{T} \delta \varphi_{j}
$$

here, the sizes of arrays $\delta \varphi_{i}, \delta \varphi_{j}$ should be the same.

The estimates $K^{\prime}\left(\delta \varphi_{i}\right)$ and $K^{\prime}\left(\delta \varphi_{i}, \delta \varphi_{j}\right)$ of covariance matrices are reduced into the estimates of $R^{\prime}\left(\delta \varphi_{i}\right)$ and $R^{\prime}\left(\delta \varphi_{i}, \delta \varphi_{j}\right)$ of matrices of correlation coefficients:

$$
\begin{aligned}
& R^{\prime}\left(\delta \varphi_{i}\right)=D_{i}^{-1 / 2} K^{\prime}\left(\delta \varphi_{i}\right) D_{i}^{-1 / 2}, \\
& R^{\prime}\left(\delta \varphi_{i}, \delta \varphi_{j}\right)=D_{i j}^{-1 / 2} K^{\prime}\left(\delta \varphi_{i}, \delta \varphi_{j}\right) D_{i j}^{-1 / 2}
\end{aligned}
$$

here: $D_{i}, D_{i j}$ - the diagonal matrixes of the principal diagonal members of the estimates $K^{\prime}\left(\delta \varphi_{i}\right)$ and $K^{\prime}\left(\delta \varphi_{i}, \delta \varphi_{j}\right)$ of covariance functions, respectively.

The accuracy of calculated correlation coefficients is defined by the standard deviation $\sigma_{r}$. It is evaluated according to the following formula:

$$
\sigma_{r}=\frac{1}{\sqrt{k}}\left(1-r^{2}\right),
$$

here: $k \rightarrow 8,000, r$-correlation coefficient. The maximum estimate of the standard deviation is obtained, when the value of $r$ is close to zero: in such a case, $\sigma_{r}^{\prime} \approx 0.01$, when $r \approx 0.5$, so $\sigma_{r}^{\prime} \approx 0.008$.

\subsection{The experimental equipment}

Herein, the linear shift device 8MT175-100 with a step motor 4247 produced from LTD „Standa“ is discussed upon.

The technical parameters of the system are following: the pitch of thread $-0.5 \mathrm{~mm}$; full pitch resolution -2.5 $\mu \mathrm{m}$; maximum speed $-10 \mathrm{~mm} / \mathrm{s}$; stroke $-100 \mathrm{~mm}$; horizontal load $-80 \mathrm{~N}$; vertical load $-30 \mathrm{~N}$.

For improvement of movement conditions, various lubricants are used in the kinematic pair "table - guide". The accuracy of movement of the carriage and its vibrations highly depend on the properties of the lubricant used, i.e. on its intended purpose and its viscosity.

For the investigation, two tables (Fig. 1a, b) were chosen; for them, different lubricants were used: universal Loctite 8103 and lubricant Braycote 601EF for working in vacuum.

LOCTITE 8103 is heavy duty for use on the moving parts at different speeds. It is a universal mineral grease consists of MoS2. It is used in roller bearings, socket and high stressed joints, plain, splines and and slideways subjected to heavy loads and vibration. Typically operating range for use $-30{ }^{\circ} \mathrm{C}$ to $+160{ }^{\circ} \mathrm{C}$. This grease is not recommended for use in oxygen rich systems, pure oxygen systems and should not be used as a lubricant for strong oxidizing materials.

Braycote $601 \mathrm{EF}$ is mineral grease designed to operate in high vacuum, in the presence of rocket oxidizers and fuels. It is used in satellites and other space applications. Typical applications include gears, O-rings and elastomers, ball and roller bearings and any applications where extreme environmental conditions would preclude the use of an ordinary grease. Greases such as this exhibit excellent shelf life due to their intrinsic inertness.

The principal elements of a table are shown in Fig. 2.

As it may be seen from the scheme of principal movable elements, the system operates as follows (see Fig. 1): the step motor generates a torque, the torque through the coupling is transmitted to the screw, the screw rotates inside the screw-nut (thus forming a screw pair). In such a way, it transforms rotational motion into translational motion of the screw-nut, the screw-nut through the movable part of the positioning system transmits the translational motion to the carriage that moves in the longitudinal direction along the slideway bearings.

\subsection{The test bench}

The experiment had been carried out using the linear shift system 8MT175-100 fixed to the optical table 
through an aluminium plate. The step motor and the microswitches were controlled by controller 8SMC4-USB-B8-1 (from LTD "Standa") using ILab software. The device is connected to the controller by cable with joints DB9(M) and HDB 15(M); the controller itself is connected to PC by USB cable. In addition, $36 \mathrm{~V}$ power supply block was used. For measuring vibration parameters, the means from Denmark company "Brüel \& Kjær" were used. In Fig. 2, the test bench used for investigation of dynamic properties of the positioning table, including the data collection and processing equipment 3660D with DELL computer, the three-axis accelerometer 4506 and the linear positioning table under investigation, is shown. The measurements of vibrations of the linear positioning table were carried out in three directions, for analysis used results of vertical direction measurements.

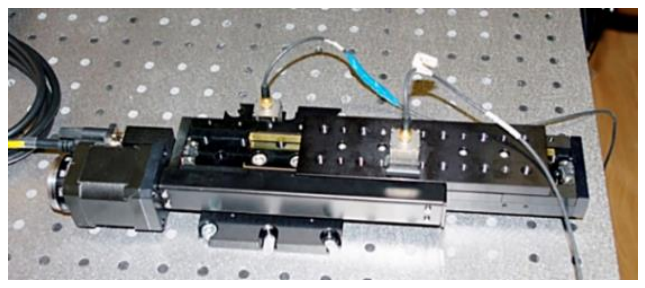

a

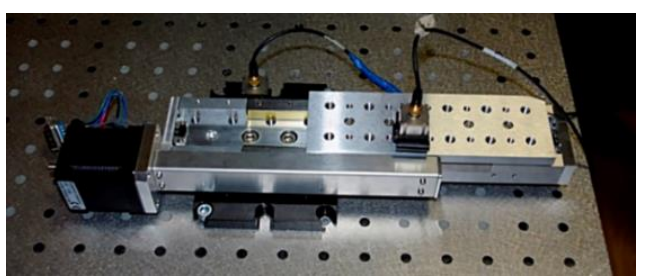

$\mathrm{b}$

Fig. 1 The scheme of principle movable elements of positioning table system 8MT175-100: a - standard linear positioning system 8MT175-100 (where lubricant Loctite 8103 is used); $\mathrm{b}$ - linear positioning device 8MT175-100 for working in vacuum (where lubricant Braycote 601EF is used)
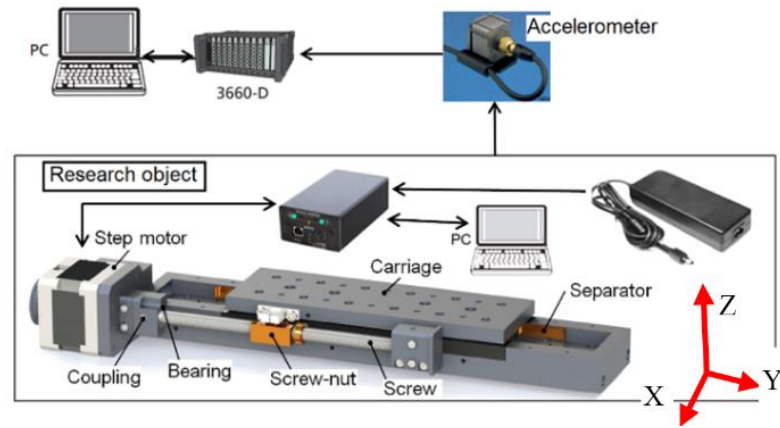

Fig. 2 The test bench for investigation of dynamic properties of positioning tables

The experiment was repeated five times to make sure about the reliability of the experimental data. The calculated statistical characteristics showed that they differ up to 5 percent. Based on this the data was taken from one measurement for further analysis. During the experiments, the speed of device 8MT175-100 was 100, 500, 1,000 and 1,500 steps per second that correspond to $0.25,1.25,2.5$ and $3.75 \mathrm{~mm} / \mathrm{s}$, respectively. To ensure a reliability of the results, the tests were repeated for five times. As it was mentioned above, in each operating mode another lubricant
(Loctite 8103 or Braycote 601EF) was used.

\subsection{The results of the measurements and their analysis}

Figs. 3-6 shows the vibrations in vertical direction ( $Z$ direction in fig. 2) of the carriages of the tables at movement speeds of $0.25,1.25,2.5$ and $3.75 \mathrm{~mm} / \mathrm{s}$. In graphs a times signals of acceleration of carriages, $b$ and $c-$ spectral graphs in which red curve - the table with the standard lubricant, the blue curve - the table with the lubricant for working in vacuum.

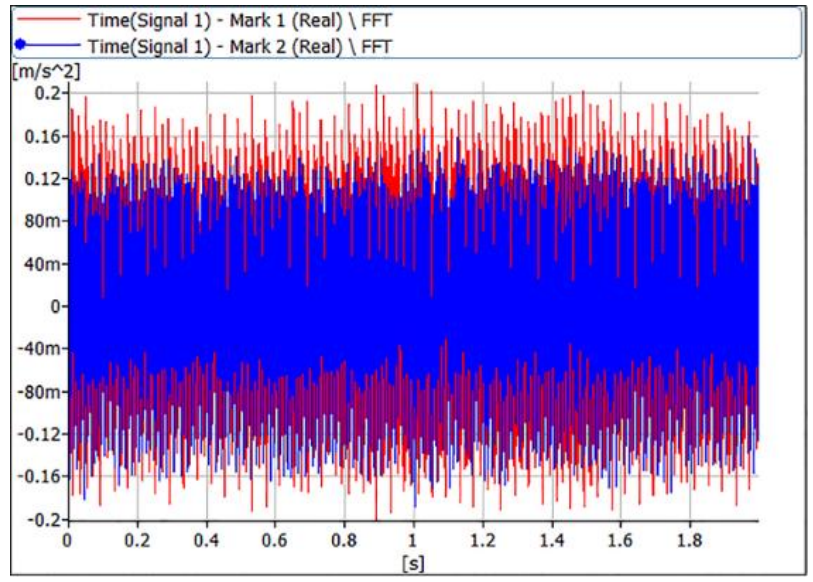

a

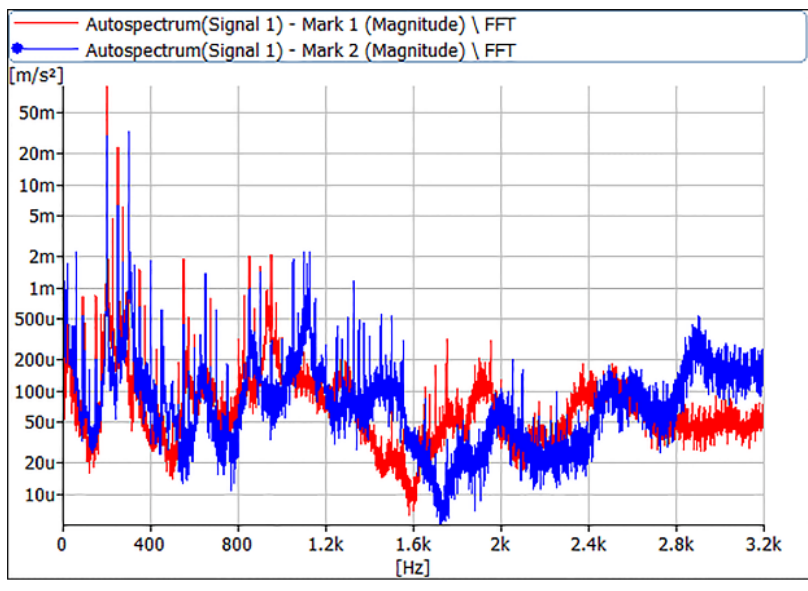

b

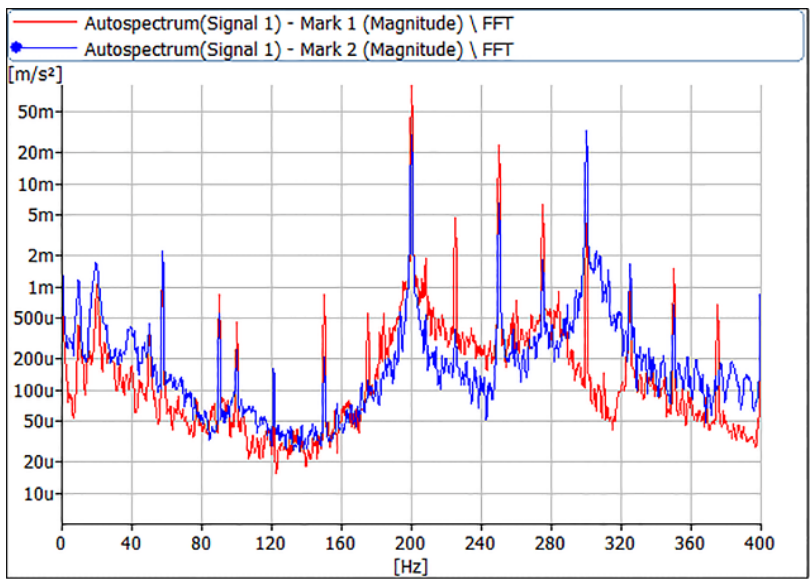

c

Fig. 3 Speed of $0.25 \mathrm{~mm} / \mathrm{s}$ (the red curve - the table with the standard lubricant Loctite 8103, the blue curve - the table with the lubricant for working in vacuum Braycote $601 \mathrm{EF}$ ), a - time signal; $\mathrm{b}$ - spectrum; $\mathrm{c}$ - spectrum extended to $400 \mathrm{~Hz}$ 
At first were compared the RMS values of a single temporal vibration signal at different lubricants. Further were compared RMS values at specific frequencies at different lubricants. As can see, the amplitudes RMS of the accelerations at $0.25 \mathrm{~mm} / \mathrm{s}$ increases by $17 \%$ when evaluating the time signal using a standard lubricant (Loctite 8103) for the carriage (Fig. 3a, red curve), this happens at a frequency of $200-280 \mathrm{~Hz}$ (Fig. 3b, c). At the speed of $1.25 \mathrm{~mm} / \mathrm{s}$, the type of lubricant does not effect to the acceleration amplitudes (Fig. 4, a). At the speed $2.5 \mathrm{~mm} / \mathrm{s}$ the accelerations amplitude decreases by $33 \%$ (Fig. 5a), this occurs at frequencies of 1,000 and $1,250 \mathrm{~Hz}$ (Fig. 5b). And at the speed $3.75 \mathrm{~mm} / \mathrm{s}$ this amplitude decreases by $17 \%$ (Fig. 6a), this occurs at frequencies 250, 1,000 and 1,250 Hz (Fig. 6b). All of these data are used for further analysis.

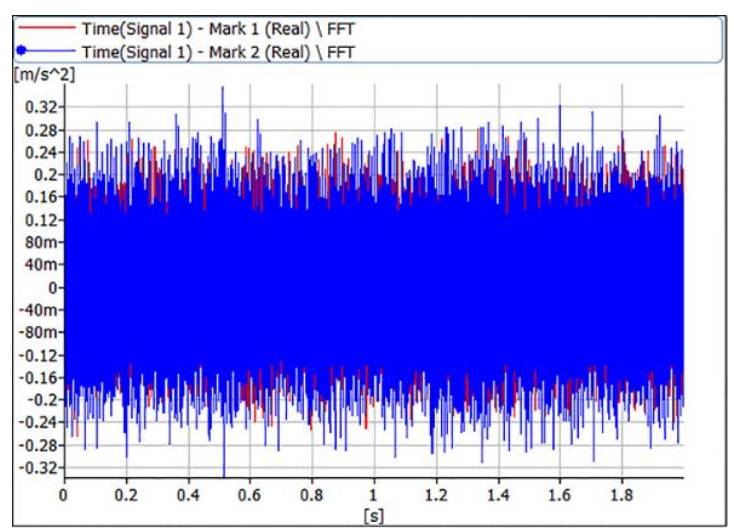

a

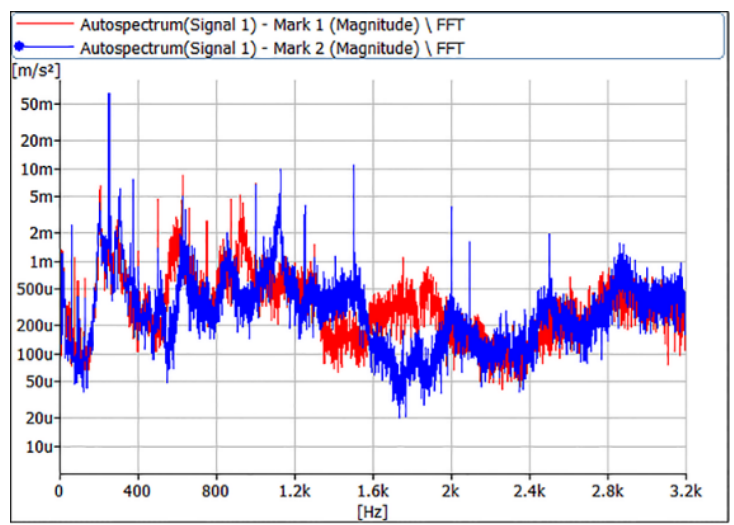

b

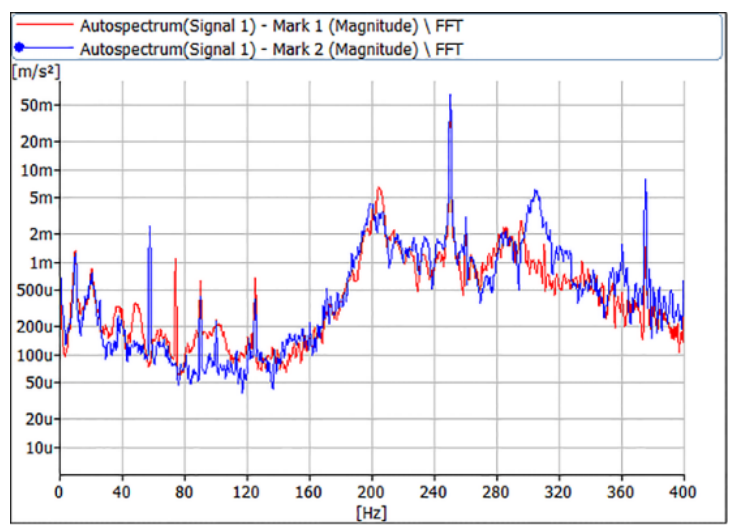

C

Fig. 4 Speed $1.25 \mathrm{~mm} / \mathrm{s}$ (the red curve - the table with the standard lubricant Loctite 8103 , the blue curve - the table with the lubricant for working in vacuum Braycote $601 \mathrm{EF}), \mathrm{a}$ - time signal; $\mathrm{b}$ - spectrum; c - spectrum extended to $400 \mathrm{~Hz}$
The vectors of vibration signals were measured in the relevant points of the positioning table and the measurement data were obtained for 4 vectors in each of two operating modes. The signals were fixed in time intervals $\tau \Delta=1.22070310 \cdot 10-4 \mathrm{~s}$ within the range of $2.0 \mathrm{~s}$. Each vector of the array included $n=16,386$ values of vibration signals.

In each operating mode, four speeds were used during the measurements and a measurement data vector was formed for each speed. So, 4 measurement data vectors were formed in each operating mode. For simplification of the vector numbering, the new numbering in the consecutive

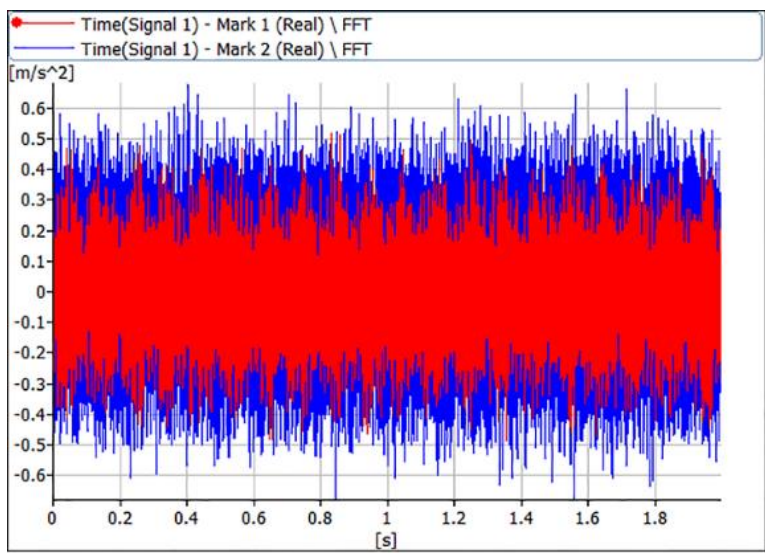

a

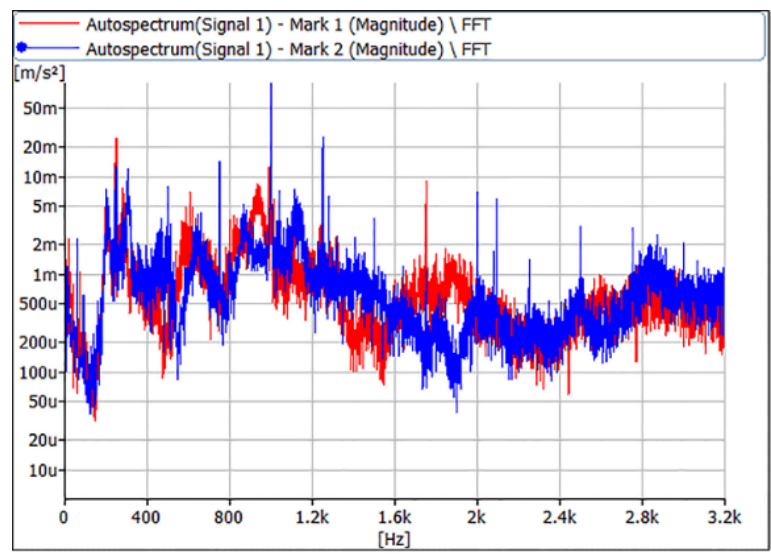

b

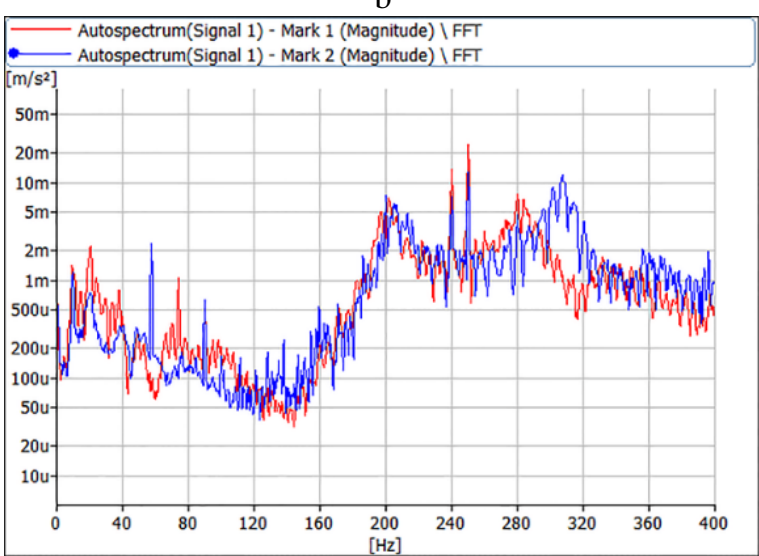

$\mathrm{c}$

Fig. 5 Speed $2.5 \mathrm{~mm} / \mathrm{s}$ (the red curve - the table with the standard lubricant Loctite 8103 , the blue crve - the table with the lubricant for working in vacuum Braycote 601EF), a - time signal; $b$ - spectrum; c - spectrum extended to $400 \mathrm{~Hz}$ 
order $(1,2,3,4)$ was used in the calculation procedures. When the vectors of both conditions were united into a single system, their numbering was $(1,2,3,4 ; 5,6,7,8)$.

The measurement data arrays were processed according to developed software upon applying operators of Matlab 7 package.

The values of the quantization interval of normed covariance functions vary from 1 to $n / 2$; here $n=16,386-$ the number of lines (values) of vectors in the array of vibration signals. The vibration signal measurement array was formed of 4 vectors (columns). For each vector, the estimate $K^{\prime}{ }_{\varphi}(\tau)$ of the normed auto-covariance function $K_{\varphi}(\tau)$ was calculated and 4 graphical expressions of normed auto-covariance functions were obtained for each mode. In addition, the estimates $K^{\prime}{ }_{\varphi}(\tau)$ of the normed cross covariance functions for vectors of all 4 points in each operating mode were calculated and 28 graphical expressions of them were obtained.

In both operating modes, the normed auto-covariance functions are the ones of periodic character. In the first operating mode, variation of estimates of the auto-covariance function depends on the speed of movement of the carriage of the positioning table. If the said speed increases, the value of the correlation coefficient $r$ decreases. In both modes, the estimates of normed auto-covariance functions rapidly "damp" from $r=$ 0 to the respective value of $r$ dependently on the speed of the carriage in the relevant operating mode (at $k=0$ ). In the first operating mode, we have the following values of correlation coefficients for 4 speeds of the carriage:

$$
\begin{aligned}
& \text { 1. }|r| \rightarrow 1 \\
& \text { 2. } r \rightarrow 1-|r| \rightarrow 0.7 \\
& \text { 3. } r \rightarrow 1-|r| \rightarrow 0.6 \\
& \text { 4. } r \rightarrow 1-|r| \rightarrow 0.2
\end{aligned}
$$

In the second operating mode, we obtain the following results for 4 speeds of the carriage:

$$
\begin{aligned}
& \text { 1. }|r| \rightarrow 1 ; \\
& \text { 2. } r \rightarrow 1-|r| \rightarrow 0.8 ; \\
& \text { 3. } r \rightarrow 1-|r| \rightarrow 0.8 ; \\
& \text { 4. } r \rightarrow 1-|r| \rightarrow 0.8 .
\end{aligned}
$$

So, the second operating mode, the speed of carriage movement inconsiderably affects the estimates of auto-covariance functions.

In a majority of cases, the estimates of normed cross-covariance functions are close to zero when $|r| \rightarrow 0.01-0.3$. However, the values or correlation coefficients are considerably higher in cases when the same speeds of the carriage are used for calculation of correlation in both operating modes. For both operating modes, it may be written:

1. at the speed 1 of the carriage $(0.25 \mathrm{~mm} / \mathrm{s})$ : the cross-correlation of signals $|r| \rightarrow 0.6$;

2 . at the speed 2 of the carriage $(1.25 \mathrm{~mm} / \mathrm{s})$ : the cross-correlation of signals $|r| \rightarrow 0.7$;
3 . at the speed 3 of the carriage $(2.50 \mathrm{~mm} / \mathrm{s})$ : the cross-correlation of signals $|r| \rightarrow 0.7$;

4 . at the speed 4 of the carriage $(3.75 \mathrm{~mm} / \mathrm{s})$ : the cross-correlation of signals $|r| \rightarrow 0.03$ (in this case, correlation is considerably less).

In addition, a considerable correlation takes place in the second mode between the signals corresponding to speeds 3 and 4 of the carriage.

The most important graphical expressions of normed auto-covariance and cross-covariance functions are provided in Figs. 7-15 below. In Fig. 16, the graphical expression of the generalized (spatial) correlation matrix of the array of 8 vectors of the positioning system in two operating modes is provided. The expression of the correlation matrices turns into blocks of 8 pyramids where the values of correlation coefficients are shown by tints of the colour spectrum. The chromatic projection of the pyramids is shown in the horizontal plane.

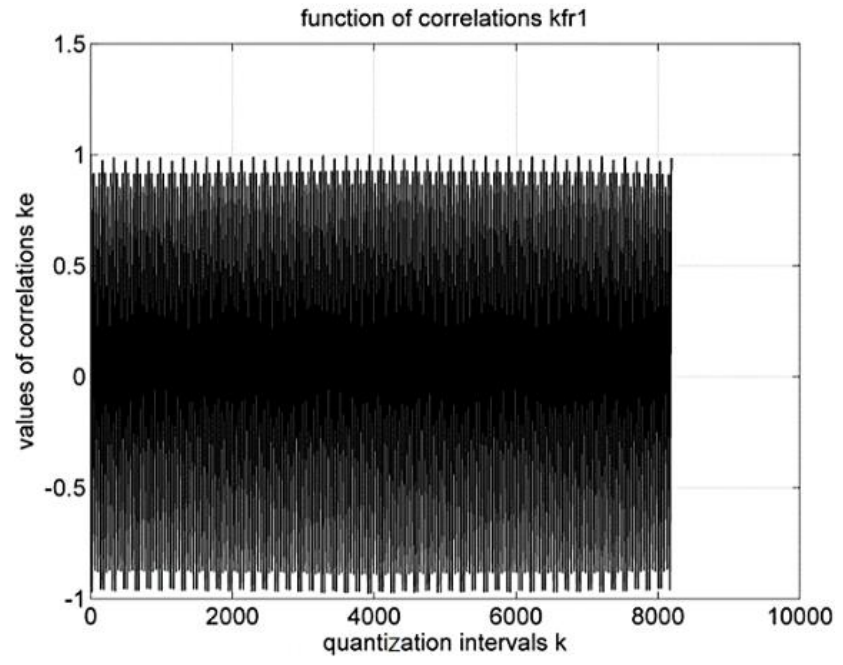

Fig. 7 The normed auto-covariance function of vibration signals at the speed 1 of the carriage in the first operating mode

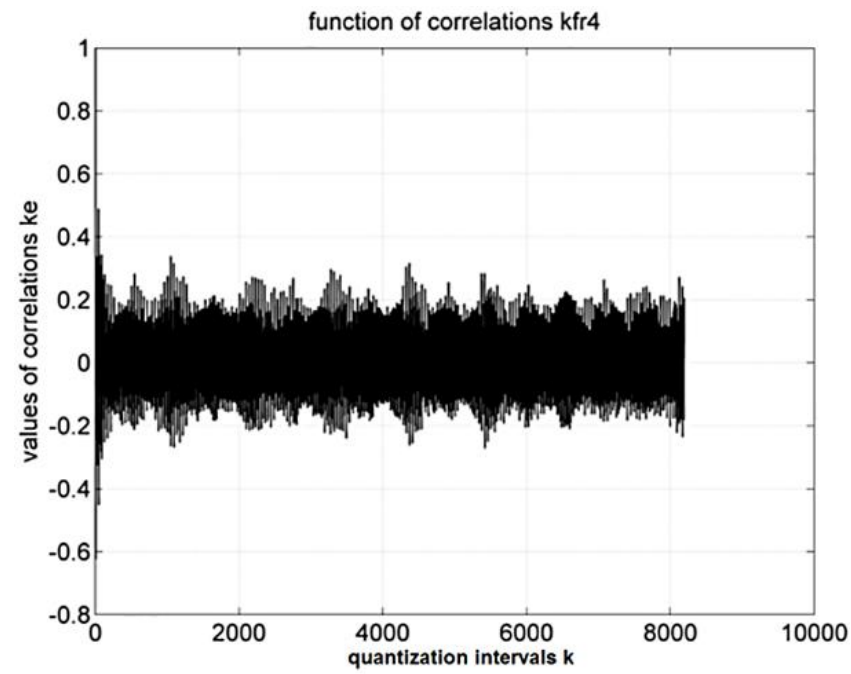

Fig. 8 The normed auto-covariance function of vibration signals at the speed 4 of the carriage in the first operating mode 


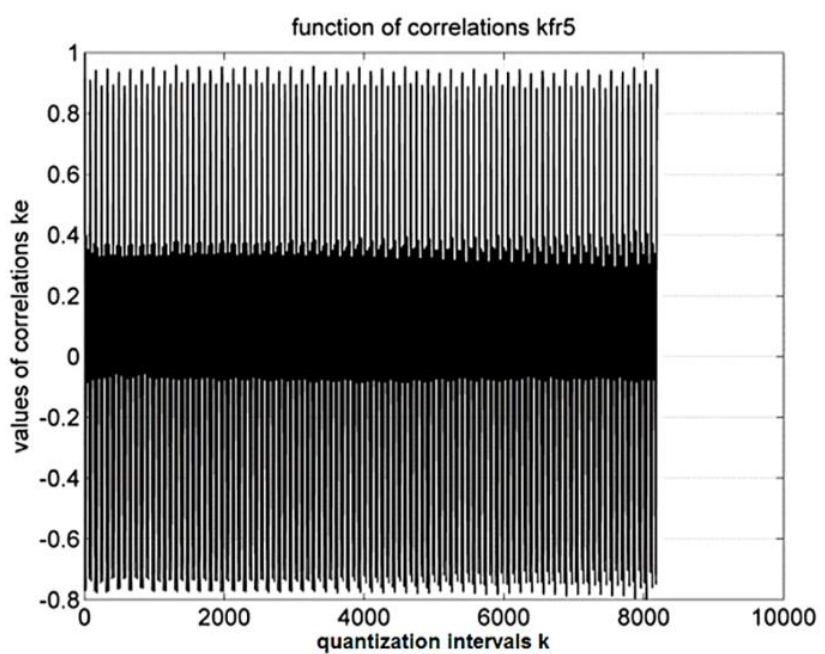

Fig. 9 The normed auto-covariance function of vibration signals at the speed 1of the carriage in the second operating mode

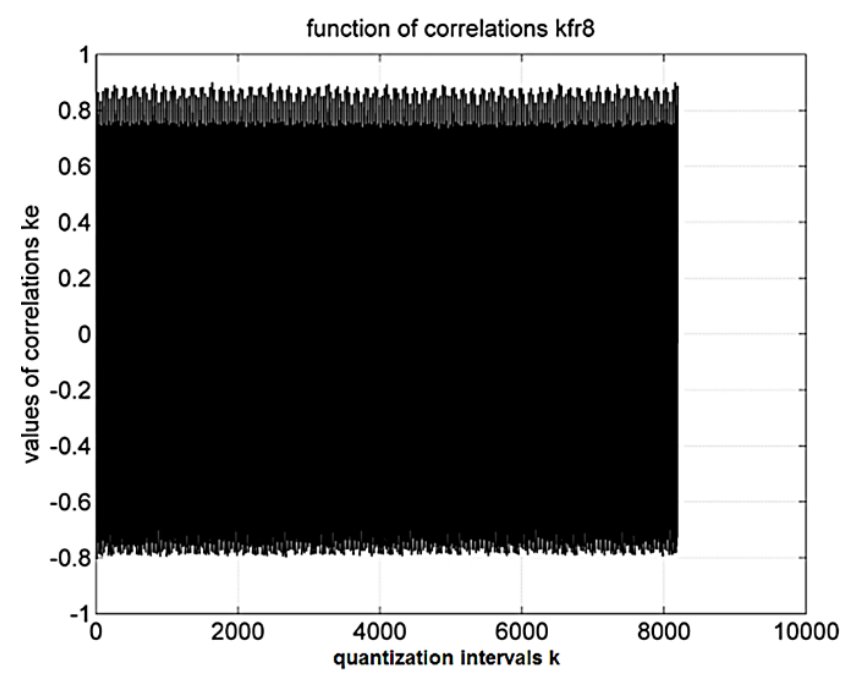

Fig. 10 The normed auto-covariance function of vibration signals at the speed 4 of the carriage in the second operating mode

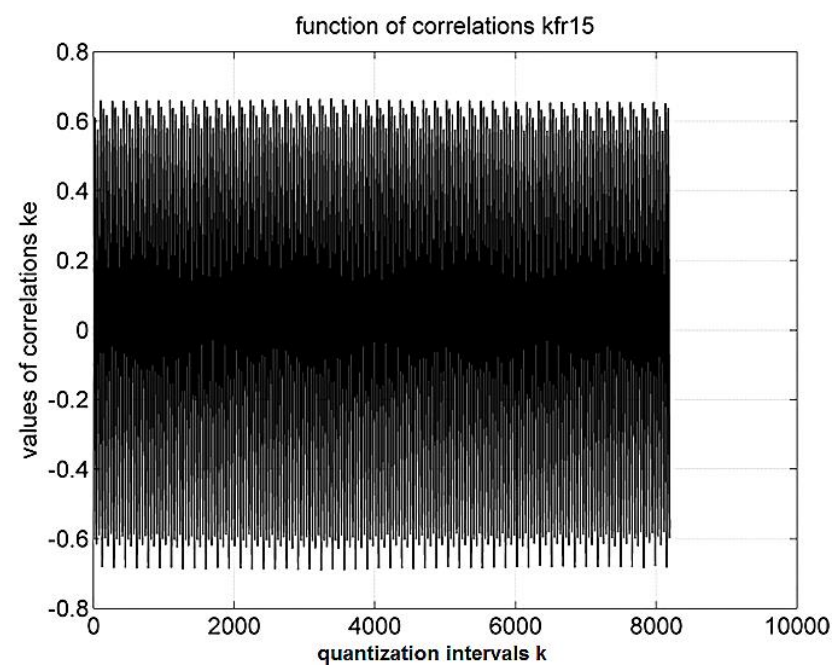

Fig. 11 The normed cross-covariance functions of vibration signals at the speed 1 of the carriage in the first and second operating modes

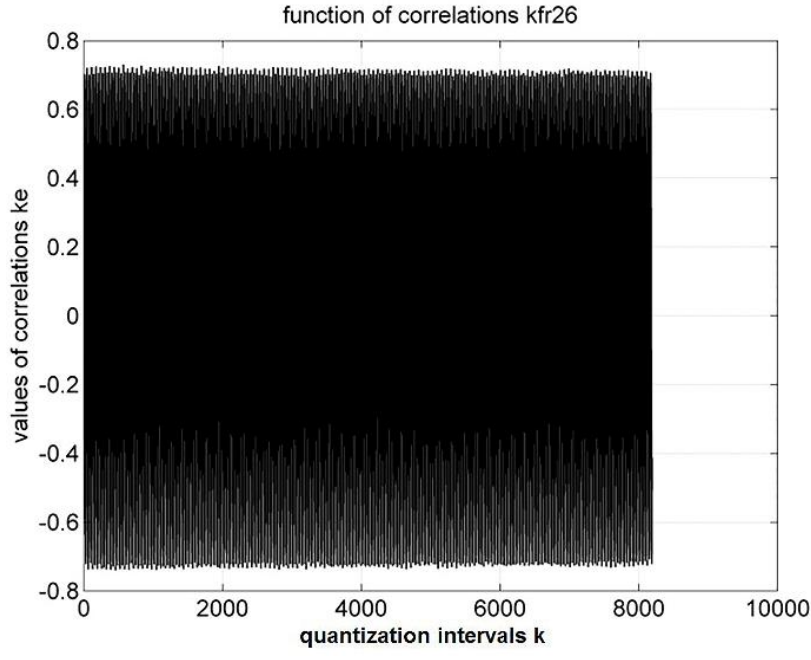

Fig. 12 The normed cross-covariance functions of vibration signals at the speed 2 of the carriage in the first and second operating modes

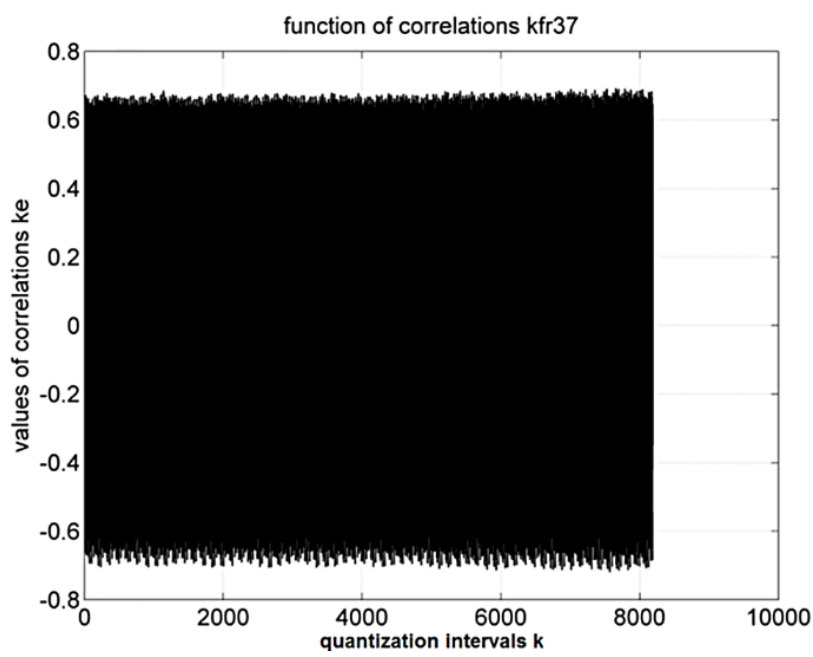

Fig. 13 The normed cross-covariance functions of vibration signals at the speed 3 of the carriage in the first and second operating modes

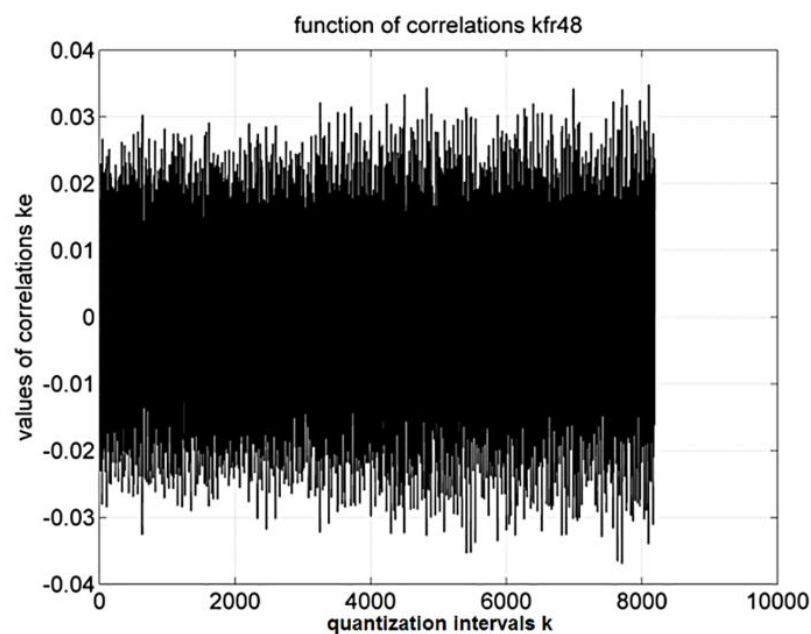

Fig. 14 The normed cross-covariance functions of vibration signals at the speed 4 of the carriage in the first and second operating modes 


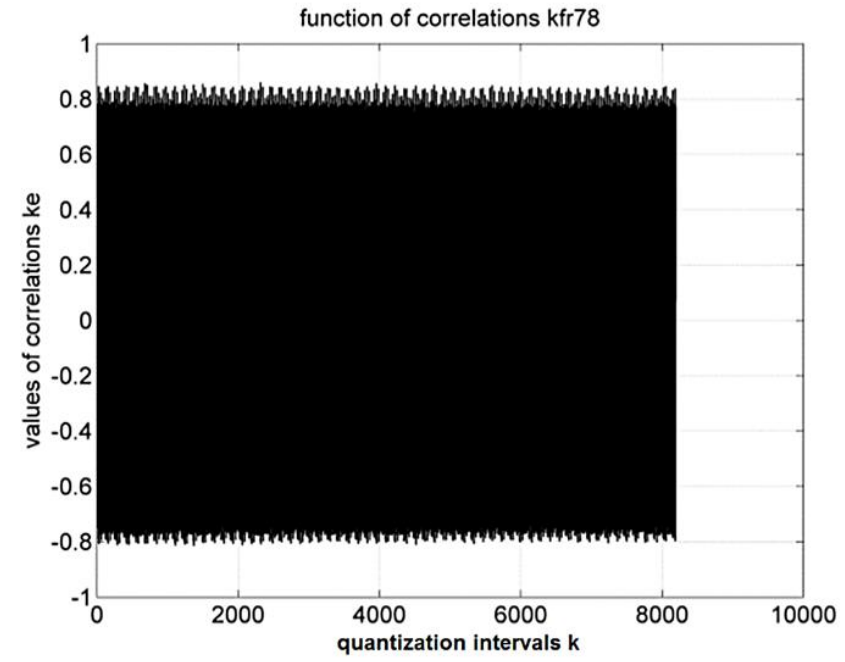

Fig. 15 The normed cross-covariance functions of vibration signals at the speeds 3 and 4 of the carriage in the second operating mode

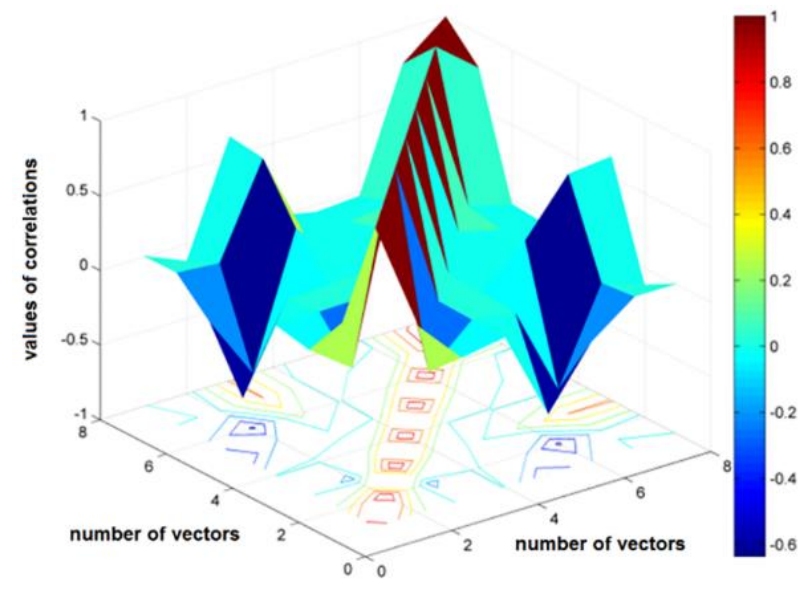

Fig. 16 The graphical expression of the generalized (spatial) correlation matrix of the array of 8 vectors of the positioning device

\section{Conclusions}

The purpose of this experimental work was to show (investigate) how different lubricants affect the vibration characteristics of the table, i.e. to investigate the vibration characteristics of the table and the correlation between operation under normal and vacuum (space) conditions.

The normed auto-covariance and cross-covariance functions of vibration signals of a linear positioning table system enable establishing changes of the correlation between the data vectors according to quantization intervals of the vibration signals. The value of correlation allows estimating the quality and accuracy of the system under analysis. In the first operating mode (where lubricant Loctite 8103 is used), variation of estimates of the auto-covariance function of vibration signals depends on the movement speed of the positioning table's carriage. If the said speed increases, the module of the correlation coefficient $r$ decreases. If the speed of the carriage is increased 15 times, the value of the correlation coefficient decreases 5 times. So, the quality and accuracy of measurements carried out by the positioning system are improved.

In the second operating mode (where lubricant
Braycote 601EF is used), the movement speed of the carriage very slightly influences the estimates of the auto-covariance functions of vibration signals. The values of correlation coefficient fall down from $|r| \rightarrow 1.0$ to $|r| \rightarrow 0.8$.

The estimates of the normed cross-covariance functions are small, when $|r| \rightarrow 0.01-0.03$ except of cases, when the same speeds of the carriage are chosen in both operating modes. In case of the same speeds of the carriage, the values of correlations coefficients between signals in both modes $|r| \rightarrow 0.6-0.7$ (practically do not depend on the speed of the carriage).

\section{References}

1. Dornfeld, D.; Min, S.; Takeuchi, Y. 2006. Recent advances in mechanical micromachining, Annals of the CIRP 55(2): 745-768.

2. Brinksmeier, E.; Mutluguenes, Y.; Klocke, F.; Aurich, J. C.; Shore, P.; Omori, H. 2010. Ultra-precision Grinding, CIRP Annals 59(2): 652-671.

3. Shore, P.; Cunnigham, C.; Debra, D.; Evans, C.; Hough, J.; Gilmozzi, R.; Kunzmann, H.; Morantz, P.; Tonnellier, X. 2010. Precision engineering for astronomy and gravity science, CIRP Annals 59(2): 694716.

4. Shinno, H.; Yoshioka, H.; Taniguchi, K. 2007. A newly developed linear motor-driven aerostatic $\mathrm{X}-\mathrm{Y}$ planar motion table system for nano-machining, Annals of the CIRP 56(1): 369-372.

5. Shinno, H.; Hashizume, H.; Yoshioka, H.; Komatsu, K.; Shinshi T.; Sat,o K. 2004. X-Y- $\theta$ Nano-positioning table system for a mother machine, Annals of the CIRP 53(1): 337-340.

6. Colby, R. S.; Mottier, F. M.; Miller, T. J. E. 1996. Vibration modes and acoustic noise in a four-phase switched reluctance motor, United Kingdom, University of Glasgow.

7. Eduardo, Rubio and Juan, Jauregui, C. 2011. TimeFrequency Analysis for Rotor-Rubbing Diagnosis, Mexico, Centro de Technologia Avanzada.

8. Ching Yuan Lin; Jui Pin Hung; Tzuo Liang Lo. 2010. Effect of preload of linear guides on dynamic characteristics of a vertical column-spindle system, Taiwan, Industrial Technology Research Institute.

9. Jui Pin Hung. 2008. Load effect on the vibration characteristics of a stage with rolling guides, Taiwan, National Chin-Yi University of Technology.

10. Liang Mi; Guo-fu Yin; Ming-nan Sun; Xiao-hu Wang. 2011. Effects of preload on joints on dynamic stiffness of a whole machine tool structure, Chengdu, Sichuan University.

11. Rodic, A.; Mester, G.; Stojković, I. 2013. Qualitative Evaluation of Flight Controller Performances for Autonomous Quadrotors, Intelligent Systems, Models and Applications, Endre Pap (Ed.), Topics in Intelligent Engineering and Informatics, 3(2), TIEI 3, http://dx.doi.org/10.1007/978-3-642-33959-2_7, Springer-Verlag Berlin Heidelberg: 115-134.

12. Mester, G.; Szilveszter, P.; Pajor, G.; Basic, D. 1994. Adaptive Control of Rigid-Link Flexible-Joint Robots, Proceedings of 3rd International Workshop of Advanced Motion Control, Berkeley, USA: 593-602.

13. Mester, G. 1995. Neuro-Fuzzy-Genetic Trajectory 
Tracking Control of Flexible Joint Robots, Proceedings of the I ECPD International Conference on Advanced Robotics and Intelligent Automation, Athens, Greece: 93-98.

14. Gamez-Montero, P.J.; Salazar, E.; Castilla, R.; Freire, J.; Khamashta, M.; Codina, E. 2009. Misalignment effects oil the load capacity of a hydraulic cylinder, International Journal of Mechanical Sciences 51: 105-113.

15. Gamez-Montero, P.J.; Salazar, E.; Castilla, R.; Freire, J.; Khamashta, M.; Codina, E. 2009. Friction effects on the load capacity of a column and a hydraulic cylinder, International Journal of Mechanical Sciences 51: $145-151$. http://dx.doi.org/10.1016/j .ijmecsci.2008.12.007.

16. Tomski, L.; Uzny, S. 2011. A hydraulic cylinder subjected to Euler's load in aspect of the stability and free vibrations taking into account discrete elastic elements, Archives of civil and mechanical engineering 11,3: 769785.

17. Uzny, S. 2009. Free vibrations and stability of hydraulic cylinder fixed elastically on both ends. Proc. Appl. Math. Mech. 9: 303-304. http://dx. doi.org 10.1002/ pamm.200910125.

18. Baragetti, S. and Villa, F. 2016. Effects of Geometrical Clearances, Supports Friction, and Wear Rings on Hydraulic Actuators Bending Behavior. Mathematical Problems in Engineering: 1-17. http://dx.doi.org/10.1155/2016/3781397.

19. Proietti T.; Luati A. 2015. The generalised autocovariance function, Journal of Econometrics 186: 245-25.

20. Siljak H.; Subasi A. 2014. Fourier spectrum related properties of vibration signals in accelerated motor aging applicable for age determination, Eksploatacja i Niezawodnosc- Maintenance and Reliability 6(4): 616621.

21. Dutkay D. E.; Jorgensen P. E. T. 2008. Fourier series on fractals: a parallel with wavelet theory, Radon Transform, Geometry, and Wavelets, Contemp. Math. 464 75101.

22. Narasimhan S.; Jordache C. 2000. Data reconciliation and gross error detection: An intelligent use of process data, Gulf Publishing Company, Huston, Texas.

23. Crowe C. M. 1996. Formulation of linear data reconciliation using information theory, Chemical Engineering Science 51(12): 3359-3366.

24. Poulin É.; Hodouin D.; Lachance L. 2010. Impact of plant dynamics on the performance of steady-state data reconciliation, Computers and Chemical Engineering 34: 354-360.

https://doi.org/10.1016/j.compchemeng.2009.11.018

25. Puigjaner L.; Heyen G. 2006. Computer aided process and product engineering, Wiley-VCH Verlag $\mathrm{GmbH}$, Weinheim, Germany, http://dx. doi.org 10.1002/9783527619856.ch1.

26. Horgan G. 1998. Wavelets for SAR image smoothing, Photogrammetric Engineering and Remote Sensing 64(12): 1171-1177.

27. Ekstrom M.; McEwen A. 1990. Adaptive box filters for removal of random noise from digital images", Photogrammetric Engineering and Remote Sensing 56(4): 453-458.

28. Antoine J. P. 2000. Wavelet analysis of signals and images, a grand tour, Revista Ciencias Matemáticas (Habana) 18: 113-143.

29. Berczynski S.; Wroblewski T. 2010. Experimental verification of natural vibration models of steel-concrete composite beams, Journal of Vibration and Control 16(14): 2057-2081. http://dx. doi.org 10.1177/1077546309350552

30. Skeivalas J.; Jurevicius M.; Kilikevicius A.; Turla V. 2015. An analysis of footbridge vibration parameters, Measurement 66: 222-228.

http://dx. doi.org 10.1016/j.measurement.2015.02.034

V. Turla, A. Kilikevicius, M. Jurevicius, A. Fursenko, K. Kilikeviciene, G. Zhetessova, O. Zharkevich

\section{ANALYSIS OF DYNAMIC PARAMETERS OF A LINEAR POSITIONING TABLE SYSTEM}

S u m m a r y

In the paper, the spread of intensity of linear positioning table system vibrations is examined and an analysis of their parameters upon applying the theory of covariance functions is carried out. For the investigation, two linear positioning tables were chosen; for them, different lubricants were used: universal lubricant Loctite 8103 and lubricant Braycote $601 \mathrm{EF}$ for working in vacuum. The results of measurements of the vibration intensity in four fixed points upon varying the speed of the carriage were recorded on the time scale in form of arrays (matrices). The estimates of cross-covariance functions of the arrays of results of measurements of the digital vibration intensity were calculated. The estimates of auto-covariance functions of single arrays upon changing the quantization interval on the time scale were calculated too. For the calculations, the software developed upon using Matlab7 package of procedures was applied.

Keywords: positioning table system, vibration signals, covariance function, quantization interval

Received July 04, 2019

Accepted October 14, 2020

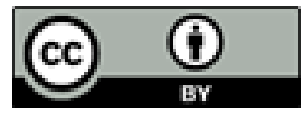

This article is an Open Access article distributed under the terms and conditions of the Creative Commons Attribution 4.0 (CC BY 4.0) License (http://creativecommons.org/licenses/by/4.0/). 